ESAIM: PROCEEDINGS, August 2010, Vol. 30, p. 124-141

D. Bresch, V. Calvez, E. Grenier, P. Vigneaux \& J.-F. Gerbeau, Editors

\title{
MUCUS DYNAMICS SUBJECT TO AIR AND WALL MOTION*
}

\author{
S. Enault ${ }^{1}$, D. Lombardi ${ }^{2}$, P. Poncet ${ }^{3}$ And M. Thiriet ${ }^{4}$
}

\begin{abstract}
This study presents a numerical investigation of basic interactions between respiratory mucus motion, air circulation and epithelium ciliated cells vibration. One focuses on identification of meaningful rheological parameters, physiological and numerical simulation dimensioning. These preliminary results are crucial before the study of more general configurations of respiratory mucus motion. The numerical study presented in this work aims at providing a first numerical tool able to simulate the effects of mucus mobility and its ability to carry out pathogens or to deliver aerosol therapy to membrane wall cells. Momentum diffusion is identified as the dominant effect, as expected in this micrometer scale configuration, and its associate momentum diffusion operator is shown to be extremely stiff. Furthermore, epithelium vibration is shown to be much more efficient than air circulation for mucus propulsion.

Résumé. Ce travail présente une étude numérique des interactions élémentaires entre le mucus de l'appareil respiratoire, la circulation de l'air et le mouvement vibratoire des cellules épithéliales ciliées tapissant la membrane pulmonaire. On s'intéresse en particulier à l'identification des paramètres les plus importants parmi les données rhéologiques, le dimensionnement physiologique et la configuration numérique. Ces résultats préliminaires sont cruciaux afin d'envisager l'étude de cas plus généraux de transport du mucus respiratoire. Un tel outil numérique aura pour but de quantifier la mobilité du mucus et sa capacité à expulser les pathogènes ou à délivrer un aérosol à travers membrane. La diffusion a été identifiée comme étant le phénomène dominant, comme attendu à l'échelle du micromètre, et son opérateur de diffusion associé a été observé comme étant extrêmement raide. D'autre part, il a été montré que la vibration de l'épithélium est bien plus efficace que la circulation de l'air pour assurer le transport du mucus.
\end{abstract}

\section{Context}

The present work aims at modeling and simulating motion of respiratory mucus film in lungs. Understanding mechanisms involved in mucus mobility is a key point for improving airway clearance, crucial for patients with respiratory disease. Human respiratory ducts are coated by epithelial cells that yield a carpet of cilia vibrating in the airway surface fluid to propulse the mucus stratum with entrapped particles outside the ventilatory system.

\footnotetext{
* This project NUMULUNG has been funded by INSA Toulouse, University Pierre and Marie Curie (Paris VI) and INRIA (Project REO).

${ }^{1}$ ENS Lyon, UMPA, CNRS UMR 5669, 46 allée d'Italie, F-69364 Lyon Cedex 7, France

2 Bordeaux Institute of Mathematics, CNRS UMR 5251, Univ. Bordeaux 1, 351, cours de la Libération, F-33405 Talence Cedex, France

3 Toulouse Institute of Mathematics, CNRS UMR 5219, Team MIP, Dept. GMM, INSA, 135 avenue de Rangueil, F-31077

Toulouse, France

${ }^{4}$ INRIA Project REO, Lab. Jacques-Louis Lions, CNRS UMR 7598, Univ. P. et M. Curie, Boite courrier 187, F-75252 Paris Cedex 5, France
}

(C) EDP Sciences, SMAI 2010 


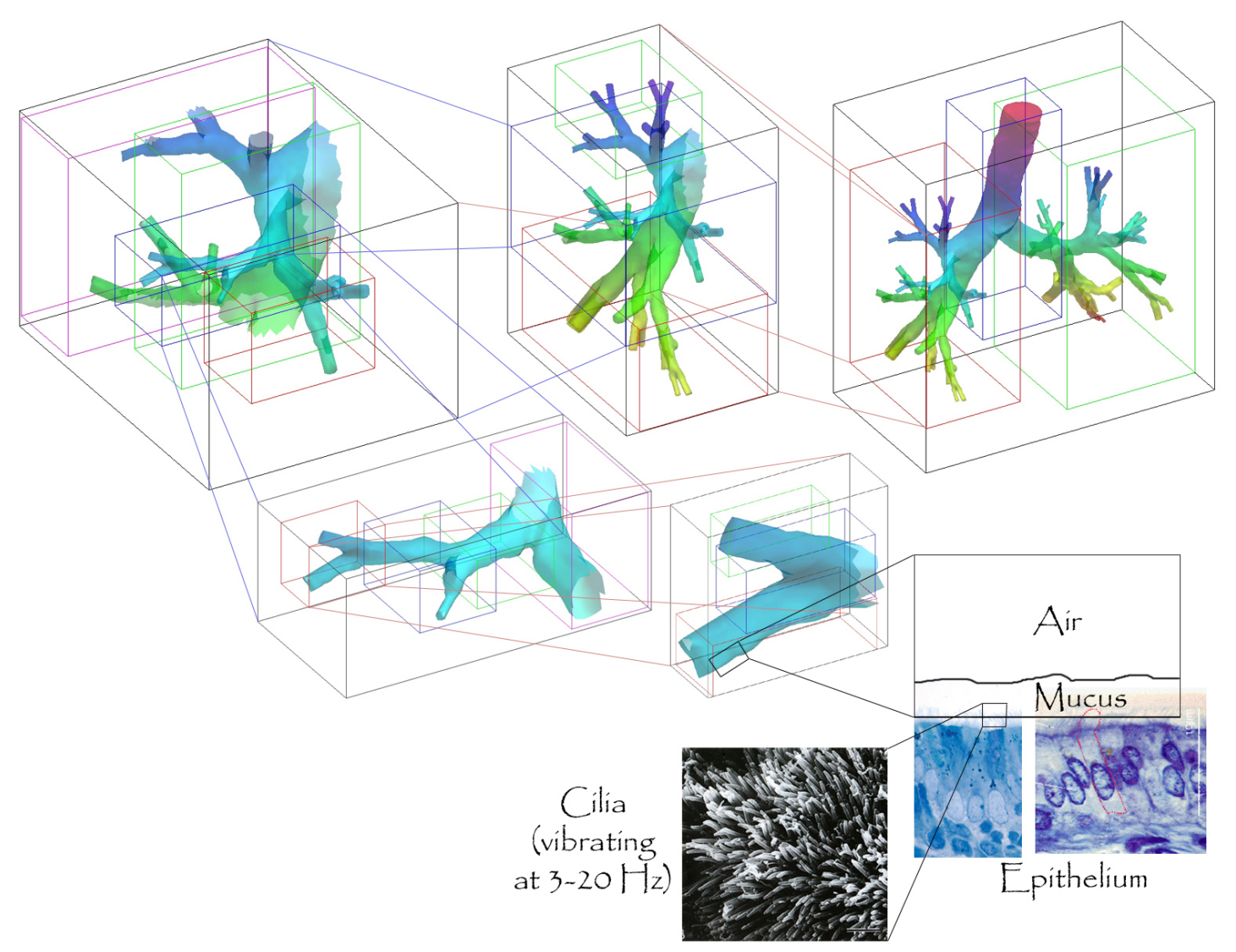

Figure 1. Real lung geometry and membrane neighborhood with air/mucus/epithelium configuration.

Mucus is a visco-elastic fluid secreted by the respiratory epithelium (see figure 1) that protects tracheo-bronchial tree mucosa from dehydration and traps inhaled particles (allergens, carcinogens, dust, micro-organisms, and inflammatory debris) that come into contact with it. Ciliary motions that are associated with mucus propulsion in the human respiratory tract cleans airways, as it flows from either the tracheo-bronchial tree or upper airways toward the pharynx, where it is swallowed (or expectorated). Strong mucus mobility is necessary to provide satisfying airway clearance and healthy behavior of respiratory system. Otherwise either proliferation of pathogen agents is observed in stagnant mucus, either lack of mucus film let pathogens slip through pulmonary membrane. Long time targeted diseases are cystic fibrosis (or mucoviscidosis), cilio-toxic affections (chronic bronchitis, virus and/or infections), and aerosol-therapy (lung cancer). Such pathologies impact directly fluid features, such as viscosity main modulus, as shown on figure 2 .

Mucus delivery and motion are investigated in the framework of flow and transport in the respiratory tract that also include gas transport under several alternative modes of ventilation, aerosol transport, and surfactant dynamics in the terminal airways. Most - if not almost all - studies of transport in airways deal with gas convection in large respiratory ducts and diffusion in bronchioles and alveoli $[2,21]$. Experimental and numerical tests focused on unsteady 1D flow in collapsible tracheas and bronchi during forced expiration, a usual manœuvre daily used in lung function testing [22,24]. In parallel, some studies aimed at investigating tracheal deformation both experimentally [23] and numerically [4]. Results obtained from measurements and simulations were validated by in vivo observations. 3D flows in simple representative models of airways were afterward studied [7,25]. Nowadays, numerical simulations are carried out in fluid domains extracted from medical image processing [10] 


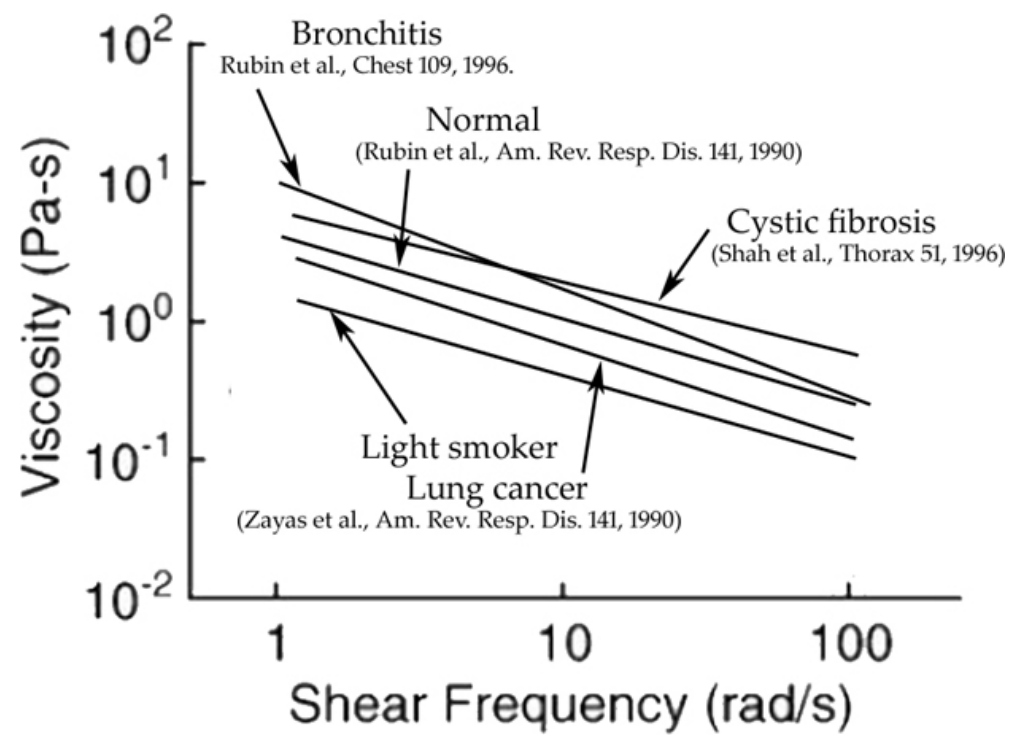

Figure 2. Different lung mucus viscosity values, altered by different pathologies, and modifying airway clearance feature (data from [13]).

and compared to in vivo measurements [19]. However, due to impaired mucus-associated respiratory pathologies as well as development of minimally invasive aerosol-based therapeutic procedures, there is a need in knowledge and simulations of mucus delivery and motion in patient-specific domains. Many attempts have been made to study separately epithelium, mucus features, and air flow in lungs, but coupling of movements of these interacting compartments has never been investigated.

Several factors have been identified to determine efficiency of mucociliary clearance: Number of cilia that synchronously beat; Cilium beating frequency; Thickness of mucus layer; Composition and rheology of mucus; Mucus thickness; Mean air velocity in bronchiole; and Geometry and gravity.

The present manuscript aims at providing a two-dimensional preliminary study on numerical simulation dimensioning, scalability, and dominant effects. Such simulations are crucial in order to predict achievable size of future three-dimensional simulations, and also to consider meaningful model for future work. Section 2 describes the fluid model, and section 3 gives problem dimensioning and boundary conditions. Discretization and numerical algorithms are described in section 4, and section 6 presents the results obtained, strategies and dominant effects.

\section{Multi-Fluid And VOrticity formulation of NAVIER-Stokes EQUATion}

The methodology used in order to model incompressible multi-fluid, is to consider one fluid with variable viscosity and density with possible jumps of these quantities. This is valid with both diffuse and sharp interfaces, and with possible extension to miscible fluids. The Navier-Stokes equations in their velocity-vorticity formulation will be used. This allows simplifying pressure from these equations and thus avoiding a special treatment for continuity of normal component of stress tensor at interface. Furthermore, in a first approach, mucus is assumed to be a Newtonian fluid.

Lagrangian velocity-vorticity methods, in three-dimensional context, have been proved to be robust, efficient and linearly scalable in complex geometry [15] under complex boundary conditions [16] with spatial [12] or temporal [12] strong variations, and even in multi-scale context [14]. Consequently, these methods offer a good framework for future work and have been chosen for the present study, in a simplified context. In order to do so, the method developed in [8] for fluid solid interaction is extended in the present work to multi-fluid. 
In the present work vorticity-velocity equations will be considered, but in their Eulerian formulation, in order to provide pure grid-based schemes and thus help implementation. Moreover, one can expect that the present microfluidic context makes the momentum diffusion stability condition much stronger than transport CFL condition, and consequently the use of Lagrangian method for this kind of simulation would be questionable.

One considers a two-dimensional domain of height $L$ and width $A$, as displayed on figure 3 . The Newton's second law of mechanics reads

$$
\frac{\partial \rho \mathbf{u}}{\partial t}+\nabla \cdot(\rho \mathbf{u} \otimes \mathbf{u})=\nabla \cdot \sigma+\mathbf{f}
$$

where $\mathbf{u}$ is the velocity field, $\rho$ the volumic mass, $\sigma=-p \mathrm{I}+\mu 2 \mathrm{D}(\mathbf{u})+\lambda(\nabla \cdot u) \mathrm{I}, \mu$ the dynamic viscosity, $p$ the pressure and $g$ the gravity, and $\mathrm{D}(\mathbf{u})=\left(\nabla \mathbf{u}+\nabla \mathbf{u}^{T}\right) / 2$ is the strain-rate tensor. Moreover, the mass conservation reads $\partial_{t} \rho+\nabla \cdot(\rho \mathbf{u})=0$. Now, two formalisms relying on vorticity will be derived from this equation.

For an incompressible fluid of constant density and viscosity, one gets the usual Navier-Stokes equations :

$$
\frac{\partial \mathbf{u}}{\partial t}+\mathbf{u} \cdot \nabla \mathbf{u}-\nu \Delta \mathbf{u}=(\mathbf{f}-\nabla p) / \rho
$$

where $\nu=\mu / \rho$. Introducing vorticity $\boldsymbol{\omega}=\nabla \wedge \mathbf{u}$ and taking the curl of equation (2) gives

$$
\frac{\partial \boldsymbol{\omega}}{\partial t}+\mathbf{u} \cdot \nabla \boldsymbol{\omega}-\boldsymbol{\omega} \cdot \nabla \mathbf{u}-\nu \Delta \boldsymbol{\omega}=0
$$

since $\nabla \wedge \nabla p \equiv 0$, under the condition that $\mathbf{f}$ derives from a potential (such as gravity). The compatibility relations are $\nabla \cdot \mathbf{u}=0$ (incompressibility), $\nabla \cdot \boldsymbol{\omega}=0$ (by definition of vorticity).

Physical boundary conditions related to the Navier-Stokes equations are often no-slip boundary conditions $\mathbf{u}=0$. When wall models are involved, non-homogeneous conditions can be considered and/or with first order conditions, such as Navier conditions for solid wall or normal stress for fluid-solid interaction and deformable walls. Virtual boundary conditions can also be periodic, no-slip-through or Neumann. In order to avoid boundary conditions on velocity while the equation is on vorticity, tangential components of boundary conditions on velocity can be converted in Neumann or Fourier-Robin conditions on vorticity [16].

Furthermore, for an incompressible fluid of variable density and viscosity, on which the external force is only gravity $\mathbf{f}=\rho \mathbf{g}$, equation (1) gives

$$
\frac{\partial \mathbf{u}}{\partial t}+\mathbf{u} \cdot \nabla \mathbf{u}-\frac{1}{\rho} \nabla \cdot(2 \mu \mathrm{D}(\mathbf{u}))=\mathbf{g}-\nabla p / \rho
$$

one which continuity of normal stress $\sigma \cdot n$ at interface can be applied. Taking the curl of this last equation gives

$$
\frac{\partial \boldsymbol{\omega}}{\partial t}+\mathbf{u} \cdot \nabla \boldsymbol{\omega}-\boldsymbol{\omega} \cdot \nabla \mathbf{u}-\nabla \wedge\left(\frac{1}{\rho} \nabla \cdot(2 \mu \mathrm{D}(\mathbf{u}))\right)=\frac{1}{\rho^{2}} \nabla \rho \wedge \nabla p
$$

since $\nabla \wedge \nabla p=0$ and $\nabla \wedge \mathbf{g}=0$. Since $\nabla p$ is defined through equation (4), equation (5) can be written

$$
\frac{\partial \boldsymbol{\omega}}{\partial t}+\mathbf{u} \cdot \nabla \boldsymbol{\omega}-\boldsymbol{\omega} \cdot \nabla \mathbf{u}-\nabla \wedge\left(\frac{1}{\rho} \nabla \cdot(2 \mu \mathrm{D}(\mathbf{u}))\right)=\frac{1}{\rho} \nabla \rho \wedge\left(\mathbf{g}-\frac{\partial \mathbf{u}}{\partial t}-\mathbf{u} \cdot \nabla \mathbf{u}+\frac{1}{\rho} \nabla \cdot(2 \mu \mathrm{D}(\mathbf{u}))\right)
$$

In Boussinesq approximation, the right hand side of equation can be reduced to $(\nabla \rho \wedge \mathbf{g}) / \rho$ (see $[8]$ for instance). This equation is valid in 3D as well as in 2D. Moreover, in the present context of two-dimensional simulations, one has no stretching, that is to say $\boldsymbol{\omega} \cdot \nabla \mathbf{u} \equiv 0$. Equation (6) is then finally reduced to

$$
\frac{\partial \boldsymbol{\omega}}{\partial t}+\mathbf{u} \cdot \nabla \boldsymbol{\omega}-\nabla \wedge\left(\frac{1}{\rho} \nabla \cdot(2 \mu \mathrm{D}(\mathbf{u}))\right)=\frac{1}{\rho} \nabla \rho \wedge \mathbf{g}
$$



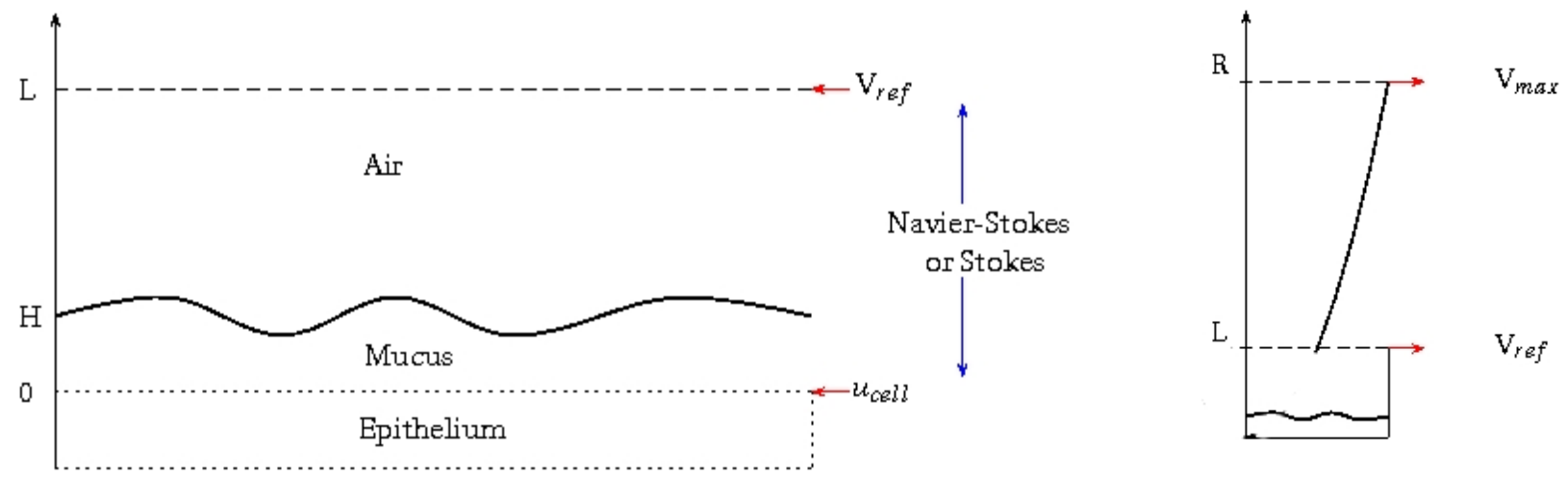

FIguRE 3. Setting of the model : Computational box and related boundary conditions (to the left), and Poiseuille flow setup for reference velocity at the top of the box (to the right).

The full governing equations, involving this last one, viscosity and density transport and boundary conditions are displayed in equation (15) of section 4. Transport is kept in the model since it can be non neglectible under some circumstances, such as strong velocities at boundaries or strong alteration of air/mucus interface topology [9].

Furthermore, in case of jumps of $\mu$ and $\rho$, calculus has to be performed carefully since multiplication of Dirac and non-continuous functions may occur. Two arguments are required to have rigorous expression of diffusion terms. On the one hand, density and viscosity can jump only together (at the fluid interface) and are both affine function of a level-set (characteristic of species, see equation (17) of section 4), so $\rho^{-1} \nabla \mu$ has the same regularity as $\mu^{-1} \nabla \mu=\nabla \ln \mu$ which are well defined as generalized function whatever the regularity of $\mu$. On the other hand, the Boussinesq approximation makes the $\mu D(\mathbf{u})$ with the same regularity as the stress $\sigma$, that is to say continuous when no surface tension is involved (the present work does not involve it in the governing equations). Consequently, $\nabla \cdot \mu D$ is a smooth generalized function (which may be singular, but without jump derivation), and $\rho^{-1} \nabla \cdot \mu D$ is well defined, even at interface.

Moreover, if future work requires on non-Boussinesq hypothesis (such as short wavelengths generated at interface), equation (5), involving $(\mathbf{u}, \boldsymbol{\omega}, p)$ models in the spirit of [18], will has to be used instead of equation (7). If surface tension is also involved, then stress is no longer continuous and more general vorticity formulations will be required in order to satisfy mathematical definition of diffusion, such as $\zeta=\nabla \times(\rho \mathbf{u})$.

\section{Dimensional AND BOUNDARY CONDITIONS SETUP}

We consider a small box of height $L$ and width $A$, as displayed on figure 3 . As the dimension of the box is negligible compared to the size of the bronchiole (in the third generation $L=8 \mu \mathrm{m}$ has to be compared to the radius $R=3 \mathrm{~mm}$ ), we can reasonably forget the curvature of the bronchiole in our model, and define differential operators in Cartesian coordinates (and not in curvilinear). In the transverse direction, we suppose periodic boundary conditions, the domain size for transverse motion being chosen as $A=2.510^{-5} \mathrm{~m}=25 \mu \mathrm{m}$ (a few epithelial cells).

Rheology is provided by the data on table 1. In the future, variable rheology of mucus will be considered in order to take into account mucus hydration, mucin hydrophilic feature and exogen ratio. Currently, mucus rheology will be considered as constant in space and time.

We set a boundary condition on the velocity $V_{\text {ref }}$, on the top virtual boundary (see figure 3 ) . Moreover, for this preliminary study, we did not consider the epithelium layer with the complex interaction between cilia, 


\begin{tabular}{cc|c|c} 
& & air & mucus \\
\hline$\rho$ & $\left(\mathrm{kg} . \mathrm{m}^{-3}\right)$ & 1.068 & 1000 \\
$\mu$ & $($ Pa.s $)$ & $19.04 \cdot 10^{-6}$ & $1-15$
\end{tabular}

TABLE 1. Mucus and air rheology parameters (assumed to be Newtonian).

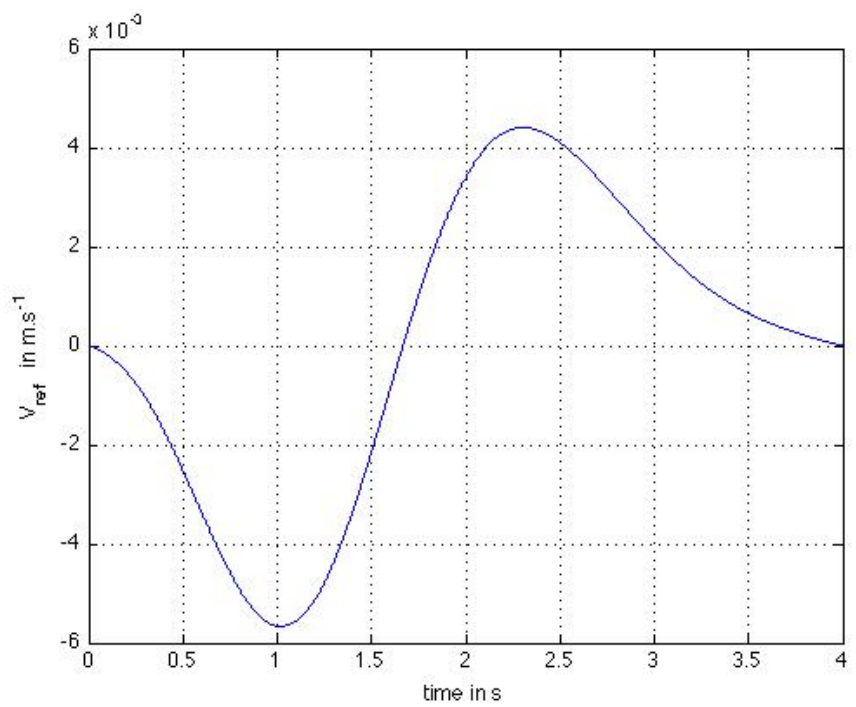

FiguRE 4. Reference velocity $V_{\text {ref }}$ during the respiratory cycle.

goblet cells, mucin and mucus. We use a virtual boundary at the bottom in the spirit of [9] (cf. figure 3), and a boundary condition on the velocity, $u_{c e l l}$, that model the output of the epithelium box.

\subsection{Top reference velocity}

Due to small size of bronchioles, air flow is supposed to be a Poiseuille flow in a cylindrical duct of radius $R$, satisfying full no-slip condition at $r=R-H, H$ being the mucus height, with mucus assumed to have a velocity neglectful toward air velocity. Indeed, this configuration leads to a small Womersley number $W o=$ $R \sqrt{2 \pi / \nu T}=0.93$, thus the frequency of pulsations is sufficiently low, so that the flow will be very nearly in phase with the pressure gradient, and will be given to a good approximation by Poiseuille's law. We can notice that a large radius or a faster respiratory cycle would lead to a state for which Poiseuille's law would not be applicable.

Maximum velocity at the center of bronchiole, assumed to be known and denoted $V_{\max }(t)$, leads to the velocity $V_{\text {ref }}$ at the top of computational box, which is the velocity at location $r=R-L$ (see figure 3 ). The reference velocity $V_{\text {ref }}$ at the top of computational box is then obtained by means of Poiseuille formula :

$$
V_{\text {ref }}(t)=v_{\text {max }}(t)\left(1-\left(\frac{R-L}{R}\right)^{2}\right) \approx \frac{2 L}{R} V_{\max }(t) \approx \frac{4 L}{\pi R^{3}} Q(t)
$$

where $Q(t)$ is the flow rate, or equivalently $V_{\max }(t)$ is the maximum velocity at the duct center. In the present configuration (typical size of third generation), $V_{\text {ref }}(t)$ (or $V_{\max }(t)$ up to a constant) is the function displayed 
on figure 4. The interpolation methodology used to obtain this function $V_{\max }(t)$ is developed in the following section 3.2 .

\subsection{Maximum air velocity during respiratory cycle}

In order to model the reference velocity at the top virtual boundary, we need to find a function $f(t)$ that mimic the air flow into the lung during the respiratory cycle. In fact, the air flow function is linked to the air velocity in the lumen and if the air velocity profile is supposed to follow a Poiseuille profile, the maximum air velocity can be found through the following equation:

$$
f(t)=\int_{S} v \cdot n d x=2 \pi v_{\max }(t) \int_{0}^{R-H}\left(1-\left(\frac{r}{R}\right)^{2}\right) r d r \approx \frac{\pi}{2} R^{2} v_{\max }(t)
$$

The respiratory cycle can be split in two phases: an inspiration one during $T_{i}$ and an expiration one during $T_{e}$. In the following we suppose that our bronchal tree is perfect, that is to say the breathed volume $V_{I}^{n}$ in the bronchial of the n-th generation is linked to the breathed volume in the trachea $V_{I}$ :

$$
V_{I}^{n}=2^{-n} V_{I}
$$

We have searched the air flow function in the form

$$
f(t)=A \theta^{\prime}(t) \sin (\theta(t))
$$

In order to fit the biologic phenomenon of breath, we impose the following constraints:

(1) At time $t=0, t=T_{i}$ and $t=T_{e}+T_{i}$ the air flow is zero. So, we want $\theta(0)=0, \theta\left(T_{i}\right)=\pi$ and $\theta\left(T_{i}+T_{e}\right)=2 \pi$.

(2) The breathed volume is given $V_{I}$. That imposes the amplitude of the function: $2 A=V_{I}$.

(3) We also want to control the derivatives at the begining $\epsilon_{d}$ and end $\epsilon_{f}$ of the respiratory cycle with $\epsilon_{d}$ and $\epsilon_{f}$ close to each other.

$$
\theta^{\prime}(0)=\sqrt{\frac{\epsilon_{d}}{A}} \quad \theta^{\prime}\left(T_{i}+T_{e}\right)=\sqrt{\frac{\epsilon_{f}}{A}}
$$

We interpolate the function $\theta$ by a polynomial function of degree 4 :

$$
\theta(t)=\alpha t^{4}+\beta t^{3}+\gamma t^{2}+\delta t
$$

The previous conditions lead to a simple linear system:

$$
\left(\begin{array}{cccc}
0 & 0 & 0 & 1 \\
T_{i}^{4} & T_{i}^{3} & T_{i}^{2} & T_{i} \\
T^{4} & T^{3} & T^{2} & T \\
4 T^{3} & 3 T^{2} & 2 T & 1
\end{array}\right)\left(\begin{array}{c}
\alpha \\
\beta \\
\gamma \\
\delta
\end{array}\right)=\left(\begin{array}{c}
\sqrt{\frac{2 \epsilon_{d}}{V_{I}}} \\
\pi \\
2 \pi \\
\sqrt{\frac{2 \epsilon_{f}}{V_{I}}}
\end{array}\right)
$$

The numerical input parameters are $T=4 s, T_{e} / T_{i}=1.4$ thus $T_{i}=1.667 \mathrm{~s}$ and $T_{e}=2.333 \mathrm{~s}, V_{I}=500 \mathrm{~mL}$, which leads to the function displayed on figure 4 .

\subsection{Wall condition and cilia model}

A cilium has a planar asymmetrical motion:

- propulsion phase during a time $T_{\text {prop }}$. 

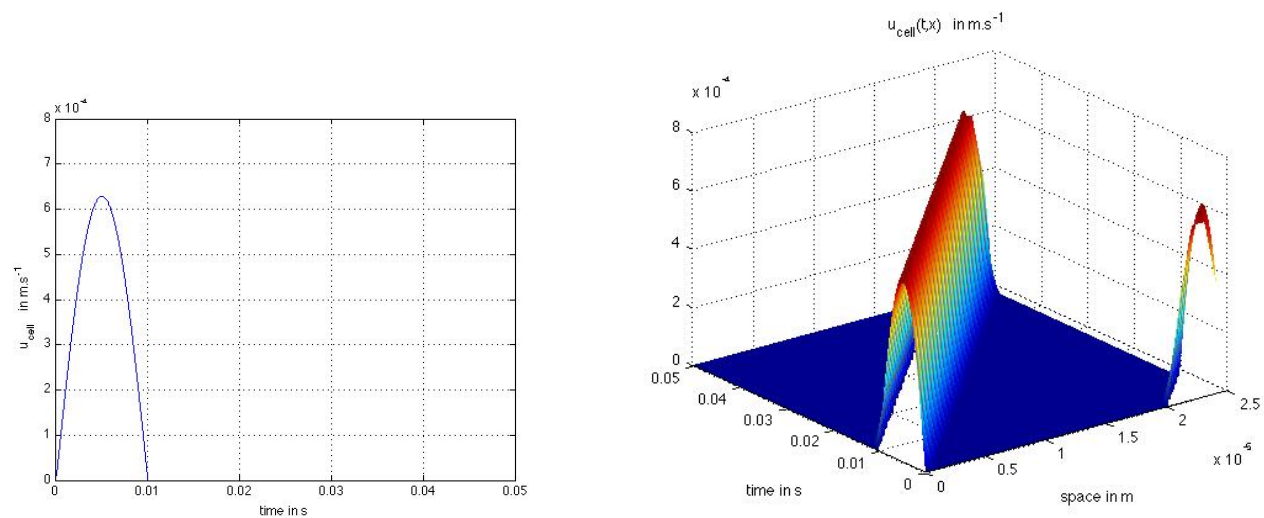

FiguRE 5. Cilium velocity with respect to time (left picture) and naive model for velocity of 10 cilia with respect to time and space (right picture).

\begin{tabular}{cc}
\hline Total time $T_{\text {tot }}$ & $50 \cdot 10^{-3} \mathrm{~s}$ \\
\hline Propulsion time: $T_{\text {prop }}$ & $10 \cdot 10^{-3} \mathrm{~s}$ \\
Rest Time $T_{\text {repos }}$ & $13 \cdot 10^{-3} \mathrm{~s}$ \\
Return time $T_{\text {retour }}$ & $27 \cdot 10^{-3} \mathrm{~s}$ \\
\hline cilium height & $7 \cdot 10^{-6} \mathrm{~m}$ \\
cilium height in the mucus & $1-2 \cdot 10^{-6} \mathrm{~m}$ \\
\hline cilium mean velocity & $70-80 \cdot 10^{-6} \mathrm{m.s}$ \\
\hline
\end{tabular}

TABLE 2. Characteristic time, space and velocity values for epithelium cilia motion.

- rest phase during a time $T_{\text {rest }}$.

- recovery phase during a time $T_{\text {return }}$ : the cilium bends while its return.

During this time, the cilium has a mean velocity $u_{m}$. We assume that during the propulsion phase, the cilium has a sinusoidal movement, and after the velocity is zero during $T_{\text {rest }}+T_{\text {return }}$.

$$
u_{\text {cell }}(t)= \begin{cases}\frac{T_{t o t} \pi u_{m}}{2 T_{\text {prop }}} \sin \left(\frac{\pi t}{T_{\text {prop }}}\right) & \text { sur } t \in\left[0, T_{\text {prop }}\right] \\ 0 & \text { sur } t \in\left[T_{\text {prop }}, T_{\text {tot }}\right]\end{cases}
$$

This leads to the curve displayed on figure 5 for cilium velocity, using values displayed on table 2 . In our simulation we use a box of 10 cilia. A model for the motion of a set of cilia is required, and we have decided to take a traveling wave of our cilium motion, so that this "kind of hola" motion could be optimal for mucus transport. Future work will require to investigate effect of cilia synchronization of mucus transport, and to build a more realistic model based on these investigations. In practice, cilia have a periodic motion with a frequency that lies between 4 and $25 \mathrm{~Hz}$; The value $20 \mathrm{~Hz}$ has been selected for our simulations.

\subsection{Non-dimensional system}

In order to improve a priori conditioning of the discretization of problem (7), the unknowns and the parameters are rescaled in the following way:

$$
\bar{x}=\frac{x}{x^{*}} \quad \bar{u}=\frac{u}{u^{*}} \quad \bar{t}=\frac{t}{t^{*}}=\frac{t u^{*}}{x^{*}}
$$


where $x^{*}$ and $u^{*}$ are the standard distance and velocity of the problem.

$$
\bar{\rho}=\frac{\rho}{\rho^{*}} \quad \bar{\mu}=\frac{\mu}{\mu^{*}}
$$

The Froud number, the dynamical pressure and the Reynolds number are introduced:

$$
F r=\frac{u^{*}}{\sqrt{g x^{*}}}, \quad \bar{p}=\frac{p}{\rho^{*} u^{* 2}}, \quad \operatorname{Re}=\frac{\rho^{*} x^{*} u^{*}}{\mu^{*}}
$$

We get, as a rescaling of equation (4) :

$$
\partial_{\bar{t}} \bar{u}+\bar{u} \cdot \bar{\nabla} \bar{u}=-\frac{1}{\bar{\rho}} \bar{\nabla} \bar{p}+\frac{\hat{e}_{g}}{F r^{2}}+\frac{1}{\operatorname{Re} \bar{\rho}} \bar{\nabla} \cdot(\bar{\mu} 2 \bar{D}(\bar{u}))
$$

where $\hat{e}_{g}$ is the unit vector of the gravitation. With these data, we found $R e \approx 4 \cdot 10^{-6}$ and $F r^{2} \approx 1,27 \cdot 10^{-2}$. It seems, as expected in most of microfluidic configurations, that the diffusion term is the most important phenomenon in the model.

\section{NUMERICAL ALGORITHM}

One considers equation (7) with boundary conditions described in the last section :

$$
\left\{\begin{array}{l}
\left.\frac{\partial \boldsymbol{\omega}}{\partial t}+\mathbf{u} \cdot \nabla \boldsymbol{\omega}-\nabla \wedge\left(\rho^{-1} \nabla \cdot(2 \mu \mathrm{D}(\mathbf{u}))\right)=\nabla \ln \rho \wedge \mathbf{g} \text { in } \Omega \times\right] 0, T[ \\
\nabla \cdot \mathbf{u}=0, \boldsymbol{\omega}=\nabla \wedge \mathbf{u} \text { in } \Omega \times] 0, T[ \\
\left.\frac{\partial \rho}{\partial t}+\mathbf{u} \cdot \nabla \rho=0 \text { and } \frac{\partial \mu}{\partial t}+\mathbf{u} \cdot \nabla \mu=0 \text { in } \Omega \times\right] 0, T[ \\
\mathbf{u}(x, 0)=\mathbf{u}_{0}(x) \text { on } \Omega \\
\rho(x, 0) \text { and } \mu(x, 0) \text { constant by pieces with respect to values of table } 1 \\
\mathbf{u}(x, t)=V_{\text {ref }}(x, t) \text { on top boundary } \\
\mathbf{u}(x, t)=u_{\text {cell }}(x, t) \text { on bottom boundary } \\
\mathbf{u}(x, t) \text { is periodic on left and right boundaries }
\end{array}\right.
$$

with aftermath non-dimensionalization. Initial mucus location is a film of height $H=L / 3$ at the bottom of the computational box.

First, we introduce a vorticity-to-velocity operator in the spirit of [15] : on a domain $\Omega=] 0, A[\times] 0, L[$, one takes at each time a vorticity field $\boldsymbol{\omega}: \Omega \rightarrow \mathbb{R}$ and gets a velocity field $\mathbf{u}: \Omega \rightarrow \mathbb{R}^{2}$.

This linear application is denoted $\mathbf{u}=\mathcal{A} \boldsymbol{\omega}$, and it is defined by means of the two following embedded Poisson equations :

$$
\begin{cases}-\Delta \Psi=\nabla \wedge \mathbf{u}=\boldsymbol{\omega} & \text { on }] 0, A[\times] 0, L[ \\ \Delta \Phi=0 & \text { on }] 0, A[\times] 0, L[ \\ \Psi\left(0, x_{2}\right)=\Psi\left(A, x_{2}\right) & x_{2} \in[0, L] \\ \Phi\left(0, x_{2}\right)=\Phi\left(A, x_{2}\right) & x_{2} \in[0, L] \\ \partial_{x_{2}} \Psi+\partial_{x_{1}} \Phi=\mathbf{u} \cdot \tau=V_{\text {ref }} & \text { on } x_{2}=L \\ \partial_{x_{2}} \Psi+\partial_{x_{1}} \Phi=\mathbf{u} \cdot \tau=u_{\text {cell }} & \text { on } x_{2}=0 \\ -\partial_{x_{1}} \Psi+\partial_{x_{2}} \Phi=u \cdot n=0 & \text { on } x_{2}=0\end{cases}
$$


whose solutions satisfy $\Psi, \Phi: \Omega \rightarrow \mathbb{R}$, and the velocity is finally computed by $\mathbf{u}=\nabla \wedge \Psi+\nabla \Phi$. For the Laplacian as well as for the derivations, centered second order finite difference schemes are used, except for fluxes at boundaries for which second order one-sided schemes are used.

Using such a vorticity-to-velocity operation $\mathcal{A}$, one can use time splitting algorithm in order to split transport, gravity and momentum diffusion in equation (15). These three steps, for which initial condition is the final value of the step performed before, are as follows :

(1) Convection - Let $\phi$ be a level-set function characteristic of mucus (that is to say of value 1 in mucus and 0 otherwise). The following transport equations are solved using centered second order finite differences in space and second-order Runge-Kutta scheme in time :

$$
\left\{\begin{array}{l}
\frac{\partial \boldsymbol{\omega}}{\partial t}+\mathcal{A} \boldsymbol{\omega} \cdot \nabla \boldsymbol{\omega}=0 \\
\frac{\partial \phi}{\partial t}+\mathcal{A} \boldsymbol{\omega} \cdot \nabla \phi=0
\end{array}\right.
$$

both in $\Omega \times] 0, T\left[\right.$. CFL condition $\delta t \leqslant \delta x \sqrt{3} /\|\mathbf{u}\|_{\infty}$ of this scheme is non restrictive compared to the one induced by diffusion. Density $\rho$ and dynamic viscosity $\mu$ are built afterward by $\rho(x)=\rho_{\text {mucus }} \phi(x)+$ $\rho_{\text {air }}(1-\phi(x))$ and $\mu(x)=\mu_{\text {mucus }} \phi(x)+\mu_{\text {air }}(1-\phi(x))$. Input/output boundary conditions on $\phi$ and $\boldsymbol{\omega}$ are periodic for left and right domain boundary, and Dirichlet conditions and imposed at top and bottom boundaries.

(2) Gravity - The following dynamical system can be integrated analytically in time and be discretized by centered second order finite difference scheme :

$$
\left.\frac{\partial \boldsymbol{\omega}}{\partial t}=\nabla \ln \rho \wedge \mathbf{g} \text { in } \Omega \times\right] 0, T[
$$

whose source is localized at fluid interface.

(3) Momentum diffusion - Over a time step $\left[t_{n}, t_{n}+\delta t\right]$, the following momentum diffusion equation has to be solved :

$$
\left.\frac{\partial \boldsymbol{\omega}}{\partial t}-\nabla \wedge\left(\rho^{-1} \nabla \cdot(2 \mu \mathrm{D}(\mathbf{u}))\right)=0 \text { in } \Omega \times\right] t_{n}, t_{n}+\delta t[
$$

with initial condition $\boldsymbol{\omega}\left(\cdot, t_{n}\right)$ given as the final value of gravity step. In order to avoid three successive numerical differentiations, one prefers to introduce a Stokes equation whose initial condition is $\mathbf{u}\left(\cdot, t_{n}\right)=$ $\mathcal{A} \omega\left(\cdot, t_{n}\right):$

$$
\left.\frac{\partial \mathbf{u}}{\partial t}-\frac{1}{\rho} \nabla \cdot(2 \mu \mathrm{D}(\mathbf{u}))+\nabla \gamma=0 \text { in } \quad \Omega \times\right] t_{n}, t_{n}+\delta t[
$$

since $\rho$ is stationary over this sub-step (thus $\partial \rho / \partial t \equiv 0$ ), with $\mathbf{u}=V_{\text {ref }}, \mathbf{u}_{\text {cell }}$ or periodic boundary conditions. Relation $\boldsymbol{\omega}\left(\cdot, t_{n}+\delta t\right)=\nabla \wedge \mathbf{u}\left(\cdot, t_{n}+\delta t\right)$ gives the final vorticity.

In the momentum diffusion step, $\gamma$ is not the pressure, and it is not used to ensure boundary conditions on $\mathbf{u}$, since most of them are already satisfied by definition of $\mathcal{A}$ : This is only as a projector on divergence free fields, and periodic and homogeneous Neumann boundary conditions are set at boundaries.

Moreover, stability condition of the momentum diffusion equation reads

$$
\operatorname{Max}\left(\frac{\mu_{\text {mucus }}}{\rho_{\text {mucus }}}, \frac{\mu_{\text {air }}}{\rho_{\text {air }}}\right) \frac{\delta t}{\delta x^{2}} \leqslant \frac{1}{2}
$$

With the rheological data considered herein (see table 1), one gets (as expected) a tiny time step $\delta t$ of around $10^{-10}$ when Euler or RK2 time integrators are used (Explicit Euler scheme gives a CFL at $\delta t \leqslant 510^{-10}$ ). An implicit scheme is then required. In order to solve the implicit momentum diffusion step, the GMRES method is used to solve the resultant linear system (and not a fast solver for standard Poisson equation since diffusion coefficients are not constant). Simulations have shown that the time of convergence of the GMRES algorithm 
is non linear with respect to the time step. In our configurations, it seems optimal to take the time step $\delta t$ around $10^{-4}$.

Furthermore, in order to check consistency and convergence, the cilium velocity is chosen independent of $x$. As the initial condition is also independent of $x$, a first assumption is to suppose that all the functions are independent of $x$. One gets then $\partial_{y}\left(\mu \partial_{y} u_{x}\right)=0$ and this equation can be easily solved analytically:

$$
\begin{cases}u_{x}(y)=u_{\text {cell }}+\frac{C}{\mu_{\text {mucus }}} y & \text { if } y \in[0, H] \\ u_{x}(y)=v_{\text {ref }}+\frac{C}{\mu_{\text {air }}}(y-L) & \text { if } y \in[H, L]\end{cases}
$$

with

$$
C=\left(v_{\text {ref }}-u_{\text {cell }}\right)\left(\frac{H}{\mu_{\text {mucus }}}+\frac{L-H}{\mu_{\text {air }}}\right)^{-1}
$$

\section{VALidATiON}

\subsection{Non uniform decelerating Couette flow}

In this section a test case is set up in order to validate the scheme presented above. In particular the dynamics of a plane shear flow is taken into account. The domain is $[0,4] \times[0,1] \subset \mathbb{R}^{2}$, and the time is $t \in[0,1]$. The fluid is not at constant density, instead, the latter is supposed to be a function of the space coordinate. The flow being incompressible, the density is purely advected so that the mass conservation reduces to:

$$
\nabla \cdot \mathbf{u}=0 \Longrightarrow \partial_{t} \rho+\mathbf{u} \cdot \nabla \rho=0,
$$

where $\mathbf{u}=\left(u_{x}, u_{y}\right)$ is the velocity field.

Several hypothesis will be assumed in the following:

(1) the flow is invariant in $x$ direction, i.e. $\partial_{x} \circ=0$;

(2) $u_{x}(x, 0, t)=0 ; u_{x}(x, 1, t)=f(t) ; \forall x$

(3) $u_{y}(x, 0, t)=0 ; u_{y}(x, 1, t)=0$.

The above assumptions holding, let $\rho=\rho(y, t)$ be the density distribution, the conservation of momentum can be simplified and a simple diffusion problem is found:

$$
\partial_{t} u_{x}=\frac{\mu}{\rho} \Delta u_{x} .
$$

Let us observe that, since the equation for the divergence is implicitly satisfied $\left(u_{y}=0\right.$ and $\left.u_{x}=u_{x}(y, t)\right)$ and the boundary conditions are sufficient to provide the motion, no pressure gradient is needed and, as consequence, the pressure can be set to $p=0$ (or whatever constant value). Moreover, since the motion is purely parallel to the $x$ direction, applying the conservation equation lead to:

$$
\partial_{t} \rho=0 \Longrightarrow \rho=\rho(y) .
$$

We look for a separate solution so that:

$$
u_{x}(y, t)=\Theta(t) U(y) \Longrightarrow \frac{\dot{\Theta}}{\Theta}=\frac{\mu}{\rho(y)} \frac{U^{\prime \prime}}{U},
$$

where the partial derivative with respect to time is denoted with a dot while the partial derivative with respect to the space direction is denoted with a prime. 


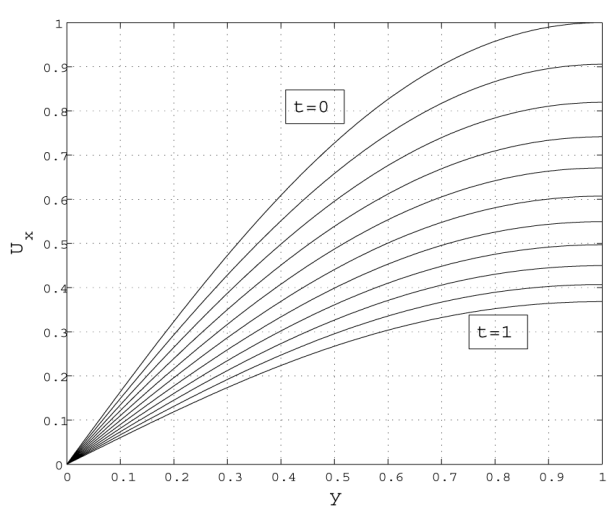

(a)

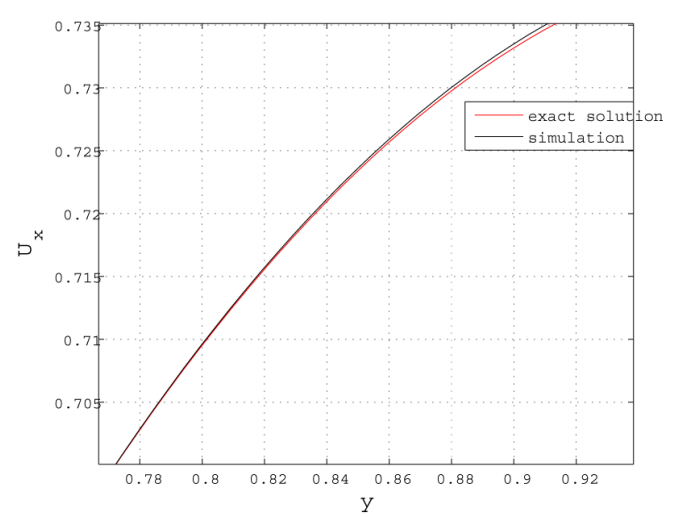

(b)

Figure 6. a) Exact solution at different times, b) Exact and numerical solution at $\mathrm{T}=0.3$, zoom.

Let us introduce the separation constant $\lambda$. The problem can be splitted into two sub-problems:

$$
\dot{\Theta}=\lambda \Theta ; \quad U^{\prime \prime}=\frac{\lambda}{\mu} \rho(y) U .
$$

The first problem is a classical initial value problem and its solution is the exponential, $\lambda$ being the inverse of a charateristic time scale. The second problem is a second order Sturm-Liouville boundary value problem. Several analytical solutions can be found for different expressions of $\rho(y)$. Let us recall a classical result of mathematical physics:

$$
\phi_{n}(y)=e^{-y^{2} / 2} H_{n}(y) \Longleftrightarrow \phi_{n}^{\prime \prime}+\left(2 n+1-y^{2}\right) \phi_{n}=0,
$$

where $H_{n}$ represents the $\mathrm{n}$-th Hermite polynomial. Choosing $\mu=1, \lambda=-1, \rho=\left(3-y^{2}\right), f(t)=e^{-t}$, the following solution is obtained:

$$
u_{x}(y, t)=y \exp \left(1 / 2-t-y^{2} / 2\right) .
$$

If the initial condition for the problem is $u_{x}(y, 0)=y \exp \left(1 / 2\left(1-y^{2}\right)\right)$ the solution written above is the unique solution for the problem.

Several numerical experiments were done varying both the space and the time resolution. A krilov subspace solver (GMRES) and a biconjugate gradient stabilized solver were tried for the solution of the diffusion substep, with analogous results. In Fig.6.a) a longitudinal section $(x=0.5)$ of the exact solution is shown at different times. Let us note that the stationary solution for a variable density Couette flow is a linear profile, as in the constant density case: the non-stationary boundary conditions induce a space variation in the inertia term, making the profile curve. In Fig.6.b) a zoom of the solution at time $\mathrm{T}=0.3$ is shown, when the space resolution is $N_{x}=200, N_{y}=200$. The agreement between the solution is good, no relevant error appeared in the numerical tests.

The convergence properties of the algorithm were analyzed. In particular the $L^{2}$ norm of the error is investigated as the space resolution is varied. The error is defined as follows:

$$
E=\int_{0}^{1} e(t) d t=\int_{0}^{1}\left(\int_{\Omega}\left(U_{x}-U^{(e)}\right)^{2} d \Omega\right)^{1 / 2} d t
$$

where $U_{x}$ is the numerical solution for the $x$ component of the velocity field, $U^{(e)}$ is the exact solution.

In Fig.7.a the time evolution of the $\mathbb{L}^{2}$ space error is plotted as function of time for different resolutions. It has a maximum at approximately one fith of the simulated evolution, and it is quite small in norm. In Fig.7.b 


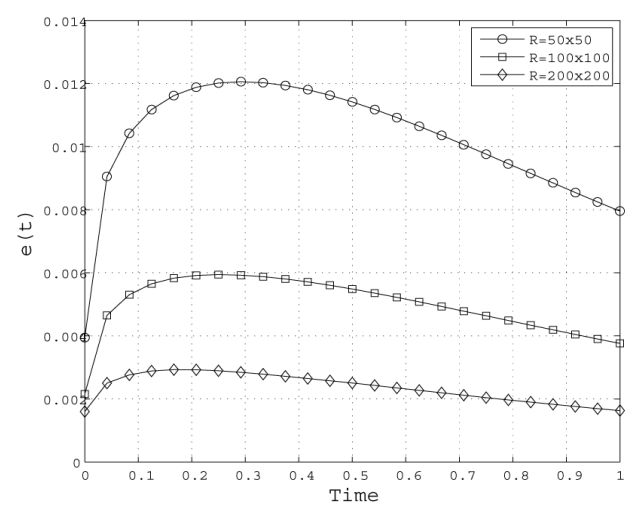

(a)

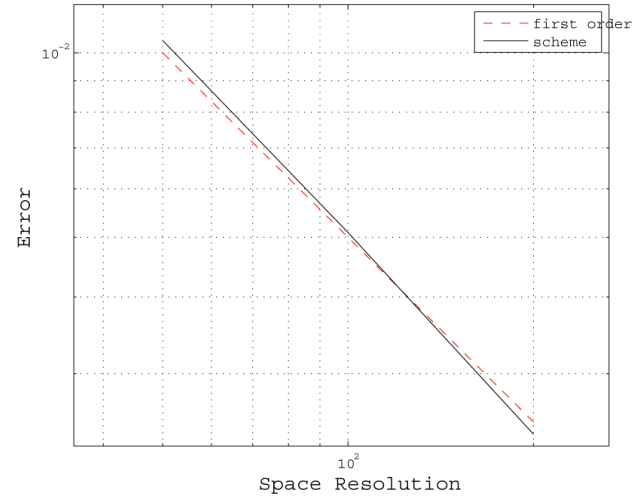

(b)

Figure 7. a) Plot of $e(t)$, where $R$ stands for the resolution; b) Logarithmic plot of $E$ as function of the space resolution.

the logarithmic plot of the error $E$ is plotted as function of the space resolution. The scheme is convergent and the convergence rate shown by the experiments results a bit faster than the first order one.

\subsection{Space varying viscosity decelerating Couette flow}

In this section a test case is set up in order to validate the scheme presented above. In particular the dynamics of a space varying viscosity Couette flow is taken into account. This flow allows us to validate the non-linear diffusion operator.

The domain is $[0, R] \times[0,1] \subset \mathbb{R}^{2}$, and the time is $t \in[0,1]$. The fluid is now considered of constant density, but the dynamic viscosity is supposed to be a function of the space coordinate. The density being constant, the mass conservation reduces to the incompressibility of the fluid:

$$
\partial_{t} \rho+\nabla \cdot(\mathbf{u} \rho)=0 \Longrightarrow \nabla \cdot \mathbf{u}=0,
$$

where $\mathbf{u}=\left(u_{x}, u_{y}\right)$ is the velocity field.

Several hypothesis will be assumed in the following:

(1) the flow is invariant in $x$ direction, i.e. $\partial_{x} \circ=0$;

(2) $u_{x}(x, 0, t)=0 ; u_{x}(x, 1, t)=f(t) ; \forall x$

(3) $u_{y}(x, 0, t)=0 ; u_{y}(x, 1, t)=0$.

The equation for the divergence leads to $\partial_{y} u_{y}=0$, and with the boundary conditions, we obtain $u_{y}(y)=0$.

The above assumptions holding, let $\nu=\nu(y)$ be the density distribution, the conservation of momentum can be simplified and a simple diffusion problem is found:

$$
\partial_{t} u_{x}-\partial_{y}\left(\nu \partial_{y} u_{x}\right)=0
$$

Let us observe that the equation for $y$ gives

$$
\rho g=\partial_{y} p
$$

thus $p=\rho g y+$ cste. We search solution $u_{x}$ as a function of separate variables:

$$
u_{x}(y, t)=\theta(t) U(y)
$$




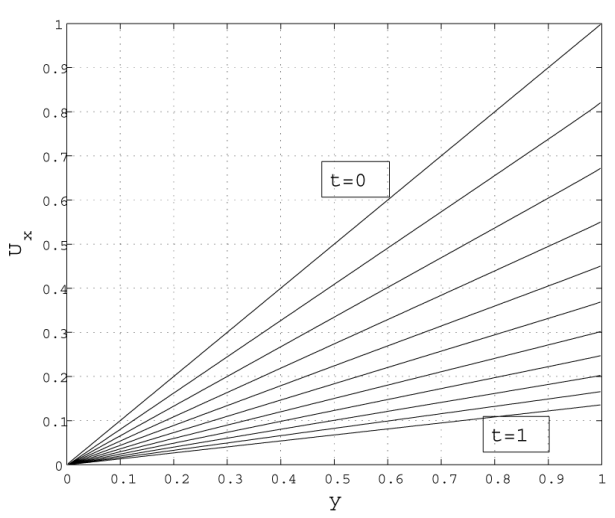

(a)

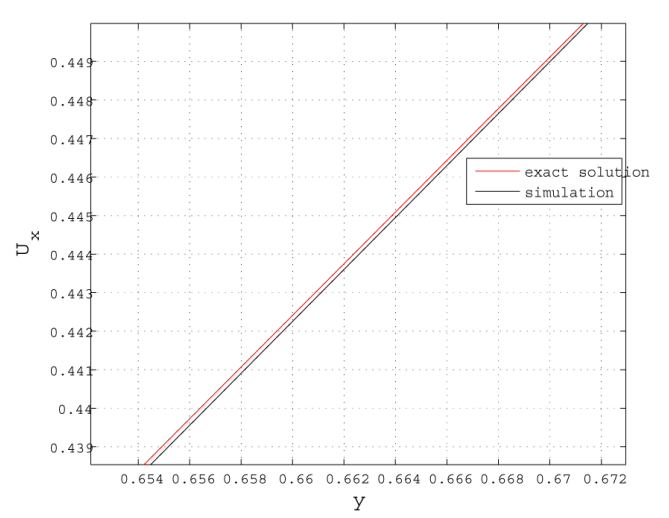

(b)

Figure 8. a) Exact solution at different times, b) Exact and numerical solution at $\mathrm{T}=0.2$, zoom.

and then we obtain:

$$
\frac{\dot{\theta}(t)}{\theta(t)}=\frac{\left(\nu U^{\prime}\right)^{\prime}}{U}=\lambda
$$

that is to say

$$
\begin{aligned}
& \theta(t)=\theta_{0} \exp (\lambda t) \propto f(t) \\
& \left(\nu U^{\prime}\right)^{\prime}-\lambda U=0
\end{aligned}
$$

Now, we notice that the $\mathrm{n}$-th Legendre polynomial $L_{n}$ is solution of :

$$
\left\{\begin{array}{l}
\left(\left(1-y^{2}\right) u^{\prime}\right)^{\prime}+n(n+1) u=0 \\
u(1)=1
\end{array}\right.
$$

When $n$ is odd, $L_{n}$ is null at the origin. We can take $\nu(y)=1-y^{2}, \lambda_{n}=-(2 n+1)(2 n+2)<0$ and

$$
\left\{\begin{array}{l}
\theta(t)=f(t)=\exp \left(\lambda_{n} t\right) \\
U(y)=L_{2 n+1}(y)
\end{array}\right.
$$

We recall some Legendre polynomials of odd order:

- $P_{1}(x)=x$,

- $P_{3}(x)=\frac{1}{2}\left(5 x^{3}-3 x\right)$

- $P_{5}(x)=\frac{1}{8}\left(63 x^{5}-70 x^{3}+15 x\right)$,

- $P_{7}(x)=\frac{1}{16}\left(429 x^{7}-693 x^{5}+315 x^{3}-35 x\right)$,

- $P_{9}(x)=\frac{1}{128}\left(12155 x^{9}-25740 x^{7}+18018 x^{5}-4620 x^{3}+315 x\right)$.

Several numerical experiments were done varying both the space and the time resolution, using the same linear algebra as in last section. In Fig.8.a a longitudinal section $(x=0.5)$ of the exact solution is shown at different times. Let us note that whether a proper exponent is chosen for the upper bound velocity deceleration, the solution is the first Legendre polynomial, scaled, i.e. it coincides with the profile of the stationary Couette flow. This is due to the fact that the momentum change induced by the deceleration is compensated by the effect of the varying viscosity. 


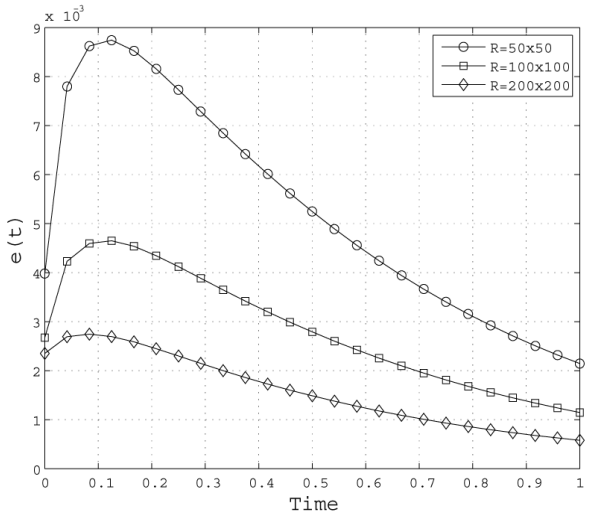

(a)

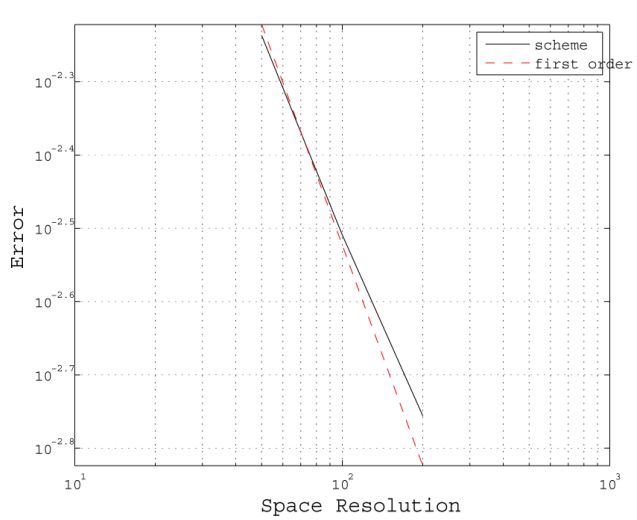

(b)

Figure 9. a) Plot of $e(t)$, where $R$ stands for the resolution; b) Logarithmic plot of $\mathrm{E}$ as function of the space resolution.
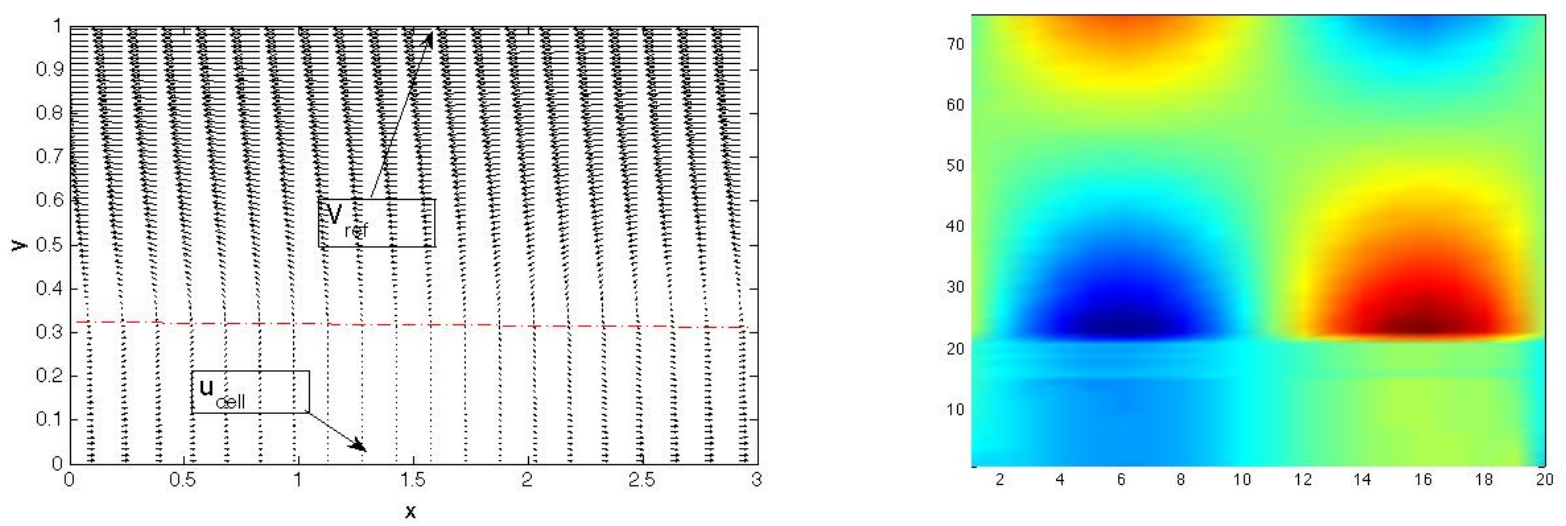

FIGURE 10. Shape of velocity with $u_{\text {cell }}(x)$ as bottom boundary condition (left picture), and resulting vorticity (right picture). Dashed line is the interface air/mucus.

In Fig.8.b a zoom of the solution at time $\mathrm{T}=0.3$ is shown, when the space resolution is $N_{x}=200, N_{y}=200$. The agreement between the solution is good, no relevant error appeared in the numerical tests. We analyzed the convergence properties, as in the previous test case. In particular the $\mathbb{L}^{2}$ norm of the error is investigated as the space resolution is varied. In Fig.9.a, the time evolution of the $\mathbb{L}^{2}$ space error is plotted as function of time for different resolutions. As in the previous case it shows a maximum at one fith of the time history. In Fig.9.b, the logarithmic plot of the error $E$ is shown. In the case of non-uniform diffusion the convergence rate is not exactly of the first order.

\section{Results}

With the algorithm described in the previous section, the parameters and boundary conditions given in section 3 , one gets that gravity and convection contribution are less than $1 \%$ : the dominant term and effect is the momentum diffusion. 


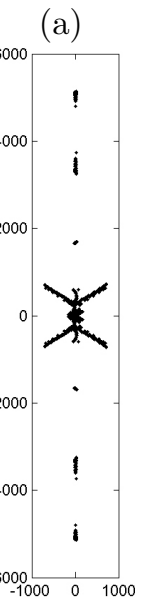

(b)

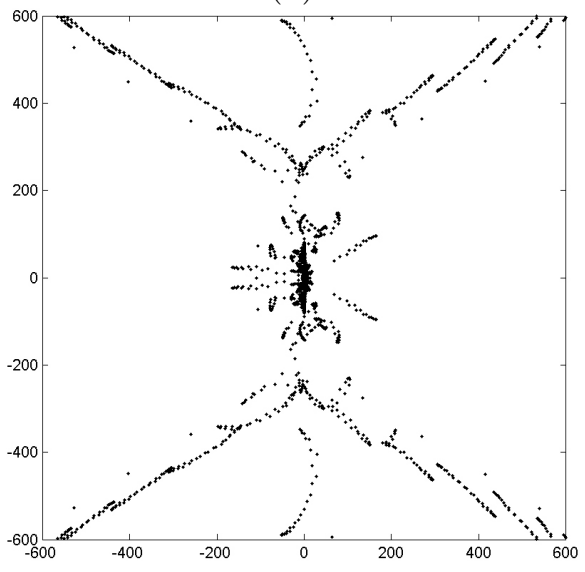

(d)

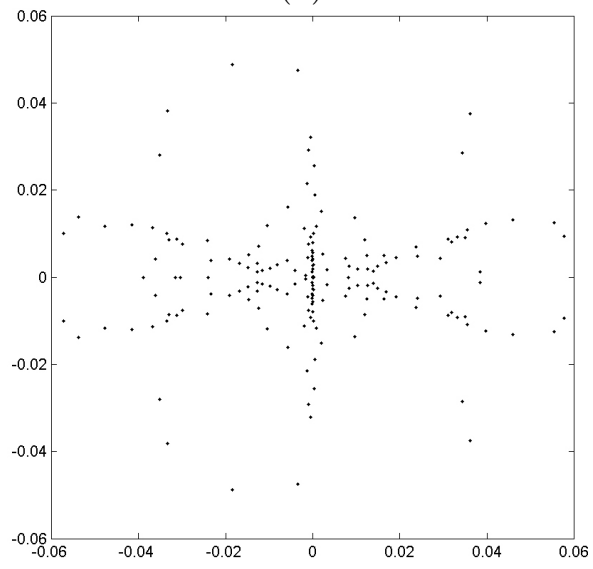

(c)

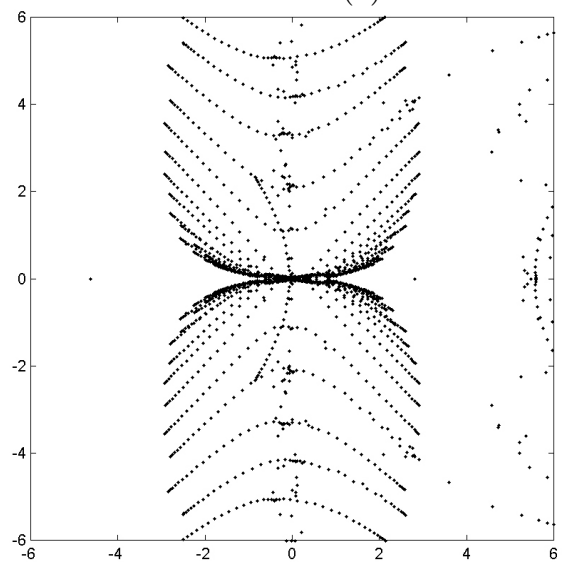

(e)

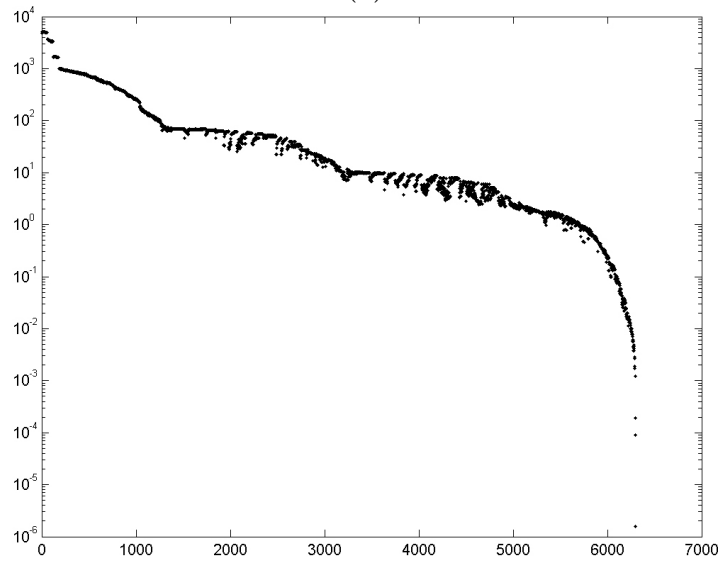

FiguRE 11. Spectrum of momentum diffusion operator, quantifying stiffness induced by momentum diffusion, with successive zoom level from top left picture (a) displaying whole spectrum, to (d) displaying zero neighborhood. Picture (e) shows eigenvalue modules.

The figure 10 is a result obtained with the first scheme. In the upper part, the velocity has a Poiseuille profile as expected. In the lower part (under the red dashed line), the shape of the velocity field is more complex: the cilium velocity field produces a vortex. The figure 10 also shows the shape of the vorticity in our numerical box : vorticity produced by the variation of cilium velocity $\mathbf{u}_{c e l l}(x)$, is diffused in the mucus almost instantly, while it propagates through the interface to the air.

At the chosen time step $\delta t=10^{-4}, 500$ time steps are required for a $20 \mathrm{~Hz}$ cilia cycle, and $410^{4}$ time steps are required for a full $4 \mathrm{~s}$ respiratory cycle. The typical spatial resolution is $\delta x_{1}=1.25 \mu \mathrm{m}$, and $\delta x_{2}=100 \mathrm{~nm}$. These setting looks like the minimum requirements for having stable and physical solutions. This will be essential for dimensioning future three-dimensional simulations.

Furthermore, a crucial point for future work is to identify the stiffness of the problem, in order to apply adequate numerical algorithms. Since it has been shown above that momentum diffusion is the dominant effect, 
focus is given on the stiffness of operator :

$$
H_{\rho, \mu}(\mathbf{u})=-\frac{1}{\rho} \nabla \cdot\left(2 \mu \frac{\nabla \mathbf{u}+\nabla \mathbf{u}^{T}}{2}\right)
$$

whose spectrum is displayed on figure 11 with several zoom levels. It depends on functions $\rho$ and $\mu$, and thus depends on air/mucus interface topology. The ratio between the largest and smallest eigenvalue modules is $510^{9}$, which means that $H_{\rho, \mu}$ is an extremely stiff momentum diffusion operator. In future, state-of-the-art "stiff" integrators will be used, such as exponential integrators and/or Krylov-based time integrators [26, 27].

Another noticeable point is that air/mucus interface remains visibly flat (deviation amplitude can be neglected toward the film height), despite the strong vorticity generation. This phenomenon would be even stronger if surface tension was involved in the model. In the present study, standard (but stable) Eulerian schemes have been chosen for solving convection and air/mucus interface transport. Such schemes have been chosen more for convenience than for their ability to track these quantities accurately. Nevertheless, the use of Lagrangian methods would help to track more accurately fluid interface and would improve accuracy of transport terms (for both vorticity and level-set defining species). On this kind of problems, motivation for Lagrangian methods is not due to stability improvement such as in aerodynamic flow simulation at higher Reynolds numbers [17].

Moreover, mucus motion follows the velocity induced by epithelium, and it is not significantly affected by air velocity (the ratio between their Reynolds numbers is 50 , see table 1 for instance).

\section{Conclusion And PeRspectives}

The present study has brought precious preliminary results on dimensioning and strategies for future threedimensional computation of mucus motion in tracheo-bonchial tree. Essential rheological parameters and characteristic lengths have been used in order to carry out minimum requirements on simulation size.

As expected, like in most microfluidic simulations, momentum diffusion is the dominant effect. The momentum diffusion operator as been shown to be extremely stiff. Adequate time integrators, such as exponential integrators, will be used in the future in order to minimize computational time of momentum diffusion.

Vorticity formulation allows to avoid management of continuity of normal stress at interface (which has to be satisfied if pressure is computed) and to consider the mucus, the pericelial mucus and the air as one fluid of sharply variable rheology. Computing accurately transport of species is then crucial in order to localize correctly mucus. In the future, more elaborate Lagrangian methods will be used for transport computing, despite the convection is not the dominant effect in global mucus motion.

The dominant input parameter has been shown to be the epithelium cilia displacement. Accurate computation of epithelium dynamics will be required in order to get correct bottom boundary conditions for mucus. Mechanical properties of cilia will also be investigated.

Moreover, the rheological model of mucus, considered as Newtonian in the present study for convenience, has to be improved in order to take into account visco-elasticity and hydration level. Visco-plasticity feature of mucus is also an open question that will also deserve attention in further works.

\section{REFERENCES}

[1] J. Adams, MUDPACK: Multigrid Fortran Software for the Efficient Solution of Linear Elliptic Partial Differential Equations, Appl. Math. Comp., 34 (1989), 113-146.

[2] L. Baffico, C. Grandmont, and B. Maury, Multiscale modelling of the respiratory tract, M3AS, 20 (2010), 59-93

[3] J. T. Beale and A. Majda, Rates of convergence for viscous splitting of the Navier-Stokes equations, Math. Comp., 37 (1981), 243-259.

[4] D. Begis et al., "A finite element model of tracheal collapse", J. Appl. Physiol. 64, 1359- 1368 (1988).

[5] A. J. Chorin, Numerical study of slightly viscous flow, J. Fluid Mech., 57 (1973), 785-796.

[6] J. P. Christiansen, Numerical solution of hydrodynamics by the method of point vortices, J. Comp. Phys. 13 (1973), 363-379.

[7] C. Conca, C. Pares, O. Pironneau, M. Thiriet, Navier-Stokes equations with imposed pressure and velocity fluxes, Int. J. Numer. Methods in Fluids 20, 267-287 (1995) 
[8] M. Coquerelle, G-H. Cottet, A vortex level set method for the two-way coupling of an incompressible fluid with colliding rigid bodies, J. Comp. Phys. 227, 9121-9137 (2008).

[9] R. V. Craster and O. K. Matar, Surfactant transport on mucus films, J. Fluid Mech., 425, 235-358 (2000).

[10] C. Fetita, S. Mancini, D. Perchet, F. Prêteux, M. Thiriet, L. Vial, An image-based computational model of oscillatory flow in the proximal part of tracheobronchial trees, Comp. Meth. Biomech. Biomed. Eng. 8(4), 279-293 (2005).

[11] L. Greengard and V. Rokhlin, A fast algorithm for particle simulation, J. Comp. Phys., 73 (1987), 325-348.

[12] P. Poncet, R. Hildebrand, G-H. Cottet and P. Koumoutsakos, Spatially distributed control for optimal drag reduction in cylinder wakes, J. Fluid Mech. 599 (2008), 111-120.

[13] S. K. Lai, Y-Y. Wang, D. Wirtz and J. Hanes, Micro- and macrorheology of mucus, Adv. Drug Del. Rev. 61, 86-100 (2009).

[14] M. El Ossmani and P. Poncet, Efficiency of multiscale hybrid grid-particle vortex methods, to appear in SIAM MMS.

[15] P. Poncet, Analysis of an immersed boundary method for three-dimensional flows in vorticity formulation, J. Comp. Phys. 228 (2009), 7268-7288.

[16] P. Poncet, Analysis of direct three-dimensional parabolic panel methods, SIAM J. Numer. Anal., 45:6 (2007), 2259-2297.

[17] P. Poncet, Topological aspects of the three-dimensional wake behind rotary oscillating circular cylinder, J. Fluid Mech. 517 (2004), 27-53.

[18] A. Riaz, E. Meiburg, Vorticity interaction mechanisms in variable-viscosity heterogeneous miscible displacements with and without density contrast, J. Fluid Mech. 517 (2004), 1-25.

[19] L. de Rochefort, L. Vial, R. Fodil, X. Matre, B. Louis, D. Isabey, G. Caillibotte, M. Thiriet, J. Bittoun, E. Durand, G. Sbirlea-Apiou, In vitro validation of CFD simulation in human proximal airways reconstructed from medical images with hyperpolarized helium-3 MRI phase contrast velocimetry, J. Appli. Physiol. 102(5):2012-2023 (2007).

[20] R. Sweet, A Parallel and Vector Variant of the Cyclic Reduction Algorithm, SIAM J. Sci. and Stat. Comp., 9 (1988), $761-766$.

[21] M. Thiriet, D. Douguet, J-C. Bonnet, C. Canonne, C. Hatzfeld, The effect on gas mixing of a He-O2 mixture in chronic obstructive lung diseases, Bull. Europ. Physio-path. Resp. 15, 1053-1068. (1982)

[22] M. Thiriet, M. Bonis, Experiments on flow limitation during forced expiration in a monoalveolar model, Med. Biol. Eng. Comput. 21, 681-687 (1983).

[23] M. Thiriet, Banc d'analyse optique de la déformation de conduites souples, Innov. Tech. Biol. Med. 8, 99-107 (1986).

[24] M. Thiriet, Experimental and theoretical models of flow during forced expiration. Pressure and pressure history dependence of flow rate, Med. Biol. Eng. Comp. 25, 551-559 (1987).

[25] M. Thiriet, R. Issa, J.M.R. Graham, A pulsatile developing flow in a bend, J. Phys. III 2, 995-1013 (1992).

[26] M. Tokman, Efficient Integration of Large Stiff Systems of ODEs with Exponential Propagation Iterative (EPI) Methods, J. Comp. Phys. 213 (2006)

[27] M. Tokman, P. Bellan, Three-dimensional Model of the Structure and Evolution of the Coronal Mass Ejections, Astronom. J. $567: 2(2003), 1202$. 